\title{
Secondary donor-derived humanized CD19- modified CAR-T cells induce remission in relapsed/refractory mixed phenotype acute leukemia after allogeneic hematopoietic stem cell transplantation: a case report
}

Meng-Yun $\mathrm{Li}^{1+}$, Zhi-Hong Lin ${ }^{2 \dagger}$, Ming-Ming Hu ${ }^{2+}$, Li-Qing Kang ${ }^{3,4+}$, Xiao-xia Wu', Qi-wei Chen ${ }^{1}$, Xin Kong ${ }^{1}$, Jian Zhang ${ }^{1 *} \mathbb{D}$, Hui-Ying Qiu ${ }^{1 *}$ and De-Pei Wu'

\begin{abstract}
Background: Mixed phenotype acute leukemia (MPAL) is a rare leukemia and is regarded as a high-risk entity with a poor prognosis. Induction therapy of an acute lymphoblastic leukemia type or hybrid regimen and hematopoietic stem cell transplantation has been recommended for MPAL. However, the optimal therapies for relapsed or refractory MPAL remain unclear, especially for relapse after stem cell transplantation. Donor-derived chimeric antigen receptor T (CAR-T) cell therapy may be a promising therapeutic option for patients with MPAL who express target antigens and have relapsed after stem cell transplantation. However, recurrence remains a challenge, and reinfusion of CAR-T cells is not always effective. An infusion of secondary donor-derived humanized CD19-modified CAR-T cells may be effective in inducing remission.

Case presentation: We report a case of MPAL with CD19 expression. The patient was treated with acute lymphoblastic leukemia-like induction and consolidation therapies but remained positive for SET-NUP214 fusion gene transcript. He subsequently underwent a haploidentical stem cell transplantation but relapsed within 6 months. He then underwent donor-derived CD19-targeted CAR-T cell therapy and achieved a sustained, complete molecular remission. Unfortunately, he developed a CD19-positive relapse after 2 years. Donor-derived humanized CD19-directed CAR-T cells induced a second complete molecular remission without severe cytokine release syndrome or acute graft-versus-host disease.

Conclusion: This case demonstrated the efficacy and safety of humanized donor-derived CD19-modified CAR-T cell infusion for treating the recurrence of MPAL previously exposed to murine-derived CD19-directed CAR-T cells.
\end{abstract}

Keywords: CD19, Chimeric antigen receptor T cells, Donor-derived, Humanized, Mixed phenotype acute leukemia

\footnotetext{
*Correspondence: crystalzj06@163.com; qiuhuiying8303@163.com

${ }^{\dagger}$ Meng-Yun Li, Zhi-Hong Lin, Ming-Ming Hu and Li-Qing Kang are co-first authors.

${ }^{1}$ Jiangsu Institute of Hematology, National Clinical Research Center for Hematologic Diseases, Collaborative Innovation Center of Hematology, The First Affiliated Hospital of Soochow University, Institute of Blood and Marrow Transplantation, Soochow University, 188 Shizi Street, Suzhou 215006, P.R. China

Full list of author information is available at the end of the article
}

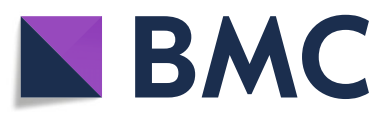

( ) The Author(s). 2020 Open Access This article is licensed under a Creative Commons Attribution 4.0 International License, which permits use, sharing, adaptation, distribution and reproduction in any medium or format, as long as you give appropriate credit to the original author(s) and the source, provide a link to the Creative Commons licence, and indicate if changes were made. The images or other third party material in this article are included in the article's Creative Commons licence, unless indicated otherwise in a credit line to the material. If material is not included in the article's Creative Commons licence and your intended use is not permitted by statutory regulation or exceeds the permitted use, you will need to obtain permission directly from the copyright holder. To view a copy of this licence, visit http://creativecommons.org/licenses/by/4.0/. The Creative Commons Public Domain Dedication waiver (http://creativecommons.org/publicdomain/zero/1.0/) applies to the data made available in this article, unless otherwise stated in a credit line to the data. 


\section{Background}

Mixed phenotype acute leukemia (MPAL) is a rare form of leukemia that constitutes only 2 to $5 \%$ of all acute leukemia, in which blasts express a complex phenotype of multiple leukemia markers from both myeloid and lymphoid lineages [1-4]. MPAL may show a B/myeloid (B/My, the most prevalent subtype), T/My, or $\mathrm{B} / \mathrm{T}$ or $\mathrm{B} /$ T/My (a rare subtype) phenotype [5]. MPAL is regarded as a high-risk entity with a poor prognosis, particularly in adults, compared with acute myelocytic leukemia (AML) and acute lymphoblastic leukemia (ALL). Optimal therapy for patients with MPAL remains controversial because of the absence of prospective clinical trials owing to the rarity of this entity. Several studies have supported a potential benefit from the application of an ALL-type or hybrid (blending elements of AML and ALL regimens) induction therapy [6-8]. Hematopoietic stem cell transplantation (HSCT) has been reported as being equally beneficial for MPAL compared with that for other acute leukemias in first or second complete remission (CR) [9-12]. However, relapse, especially after transplantation, remains a major challenge. Novel therapies, including small molecular agent, monoclonal antibody, and chimeric antigen receptor $\mathrm{T}$ (CAR-T) cell therapy, may induce another remission and longer survival. We report a case of a patient with MPAL who was previously exposed to donor-derived CD19-CAR-T cells with murine single-chain variable fragment (scFvs) for relapse after haploidentical HSCT and achieved remission with treatment by humanized CD19-CAR-T cells derived from the same donor.

\section{Case presentation}

A 29 year-old man presented to our hospital with abdominal pain and fever. Peripheral blood examination results revealed a white blood cell count of $0.56 \times 10^{9} / \mathrm{L}$, a hemoglobin level of $7.7 \mathrm{~g} / \mathrm{dl}$, and a platelet count of $43 \times 10^{9} / \mathrm{L}$. Bone marrow examination revealed an increase of blasts up to $67.2 \%$ and showed negative results for myeloperoxidase staining. Flow cytometry analysis demonstrated an abnormal blast population (48\%) expressing CD7, CD34, HLA-DR, CD10, CD19, CD33, CD117, cCD79a, and cCD3. Cytogenetic and molecular biology studies showed an abnormality of 46,XY, $\operatorname{add}(6) t(? 1 ; 6)(? p 31 ; p 24)$,del (16)(?q11)[6]/46,XY[4] and SET-NUP214 fusion gene transcript. No gene mutation was detected by next-generation DNA sequencing. These findings indicated a diagnosis of B/T MPAL with myeloid lineage expression. Induction chemotherapy with idarubicin, vincristine, and dexamethasone achieved morphological CR. Subsequently, we gave the patient consolidated treatments, including one cycle of pegaspargase combined with hyper-CVAD-B regimen (with high-dose cytarabine and methotrexate) and one cycle of
hyper-CVAD-A regimen (with cyclophosphamide, pharmorubicin, vincristine, and dexamethasone). After treatment, the patient achieved a sustained $\mathrm{CR}$, although he remained positive for the SET-NUP214 transcript as detected by quantitative real-time polymerase chain reaction. He subsequently underwent a haploidentical stem cell transplantation from his father after conditioning with the busulfan/cyclophosphamide regimen. Peripheral myeloid engraftment (absolute neutrophil count, $>0.5 \times$ $10^{9} / \mathrm{L}$ ) was evident on day 12 , and he was platelet transfusion-dependent (platelet count, $>50 \times 10^{9} / \mathrm{L}$ ) until day 14 post-HSCT. The patient sustained a complete molecular remission (CMR) until 6 months later. He then exhibited a recurrence of leukemia with the appearance of blasts (48\%) in bone marrow aspirates and a positive minimal residual disease (MRD) (85.1\%) with a phenotype of B-ALL (with expression of CD7, CD19, CD33, and cCD79a). He was positive for the SETNUP214 transcript at the time of relapse. Chimerism analysis using multiplex polymerase chain reaction to amplify an informative short tandem repeat demonstrated $51.4 \%$ donor cells. CAR-T cell therapy targeting CD19 derived from the same donor was then conducted. The CD19-directed CAR-T cells carried scFvs derived from murine antibodies. The patient underwent a fludarabine $\left(30 \mathrm{mg} / \mathrm{m}^{2}\right.$, days $\left.1-3\right)$ and cyclophosphamide (300 $\mathrm{mg} / \mathrm{m}^{2}$, days $1-3$ ) based lympho-depletion regimen before CAR-T cell infusion. The total dose was $5 \times 10^{6}$ per $\mathrm{kg}$ of body weight CAR-positive $\mathrm{T}$ cells (transduction efficiency, 34.49\%). The patient developed moderate fever twice, on days 5 and 17 after infusion, which lasted 2 and 5 days, respectively. There were no hypotension, tachycardia, hypoxia, fatigue, or life-threatening events, such as disseminated intravascular coagulation or multiorgan dysfunction after transfusion. After treatment with nonsteroidal drugs and anti-infection therapy, the patient's body temperature returned to a normal level. Additionally, a series of $\mathrm{PB}$ cytokines, including interleukin (IL)-2, IL-4, IL-6, IL-10, tumor necrosis factor, interferon- $\gamma$, and IL-17A levels, showed no significant increase; only C-reactive protein (CRP) showed a maximum fold change of 10 on day 22 (Fig. 1a). Generally, the patient's cytokine release syndrome (CRS) was grade 2. Bone marrow examination suggested CMR 9 days after CAR-T cell infusion, and the patient maintained a state of CMR combined with persistence of CD19directed CAR-T cells for 20 months without obvious development of graft-versus-host disease (GvHD) (Fig. 2, a and b). However, quantitative real-time polymerase chain reaction analysis detected that he was positive for SET-NUP214 transcript 20 months after CAR-T cell infusion. Meanwhile, a sharp decrease in bone marrow DNA copies of anti-CD19 CAR-T cells occurred (33.4 copies per microgram) (Fig. 2b). Nonetheless, the patient 
a
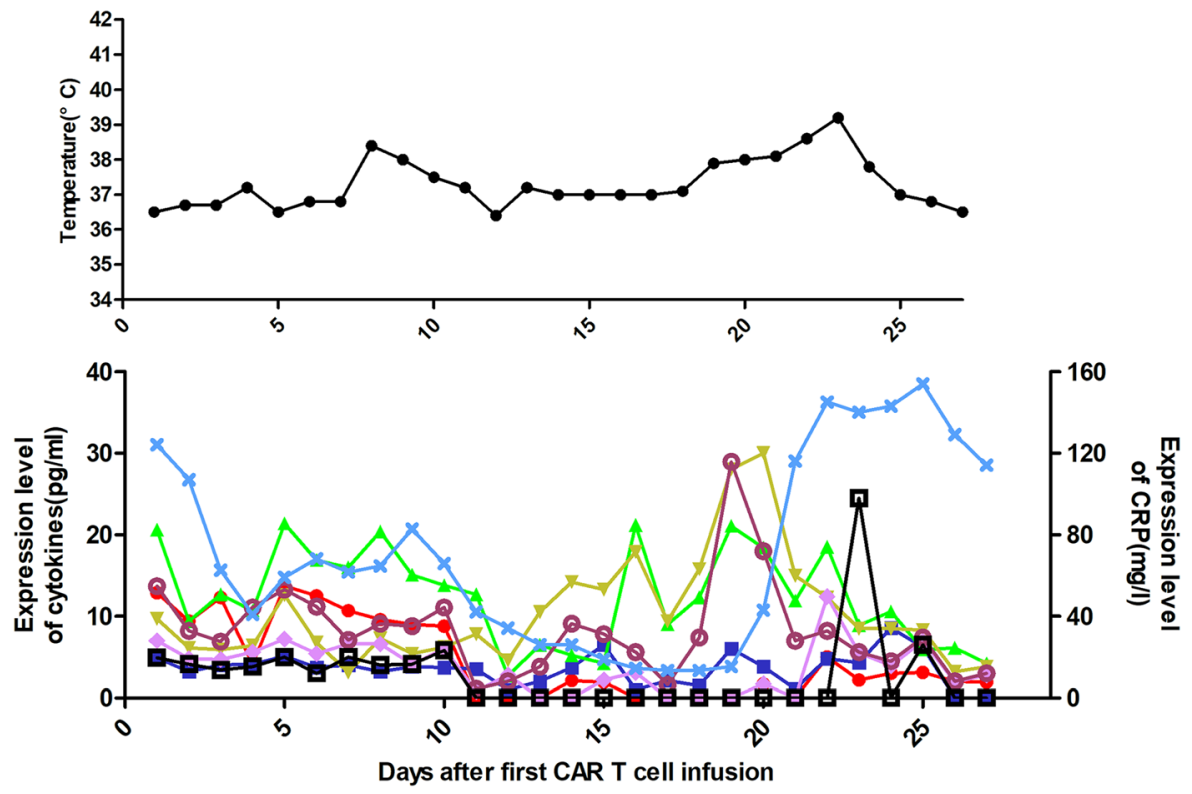

$\rightarrow \mathrm{IL}-2 \rightarrow \mathrm{IL}-4 \rightarrow \mathrm{IL}-6 \rightarrow \mathrm{IL}-10 \rightarrow \mathrm{TNF}$

$\rightarrow$ IFN-Y $\quad$ IL-17A * CRP

b
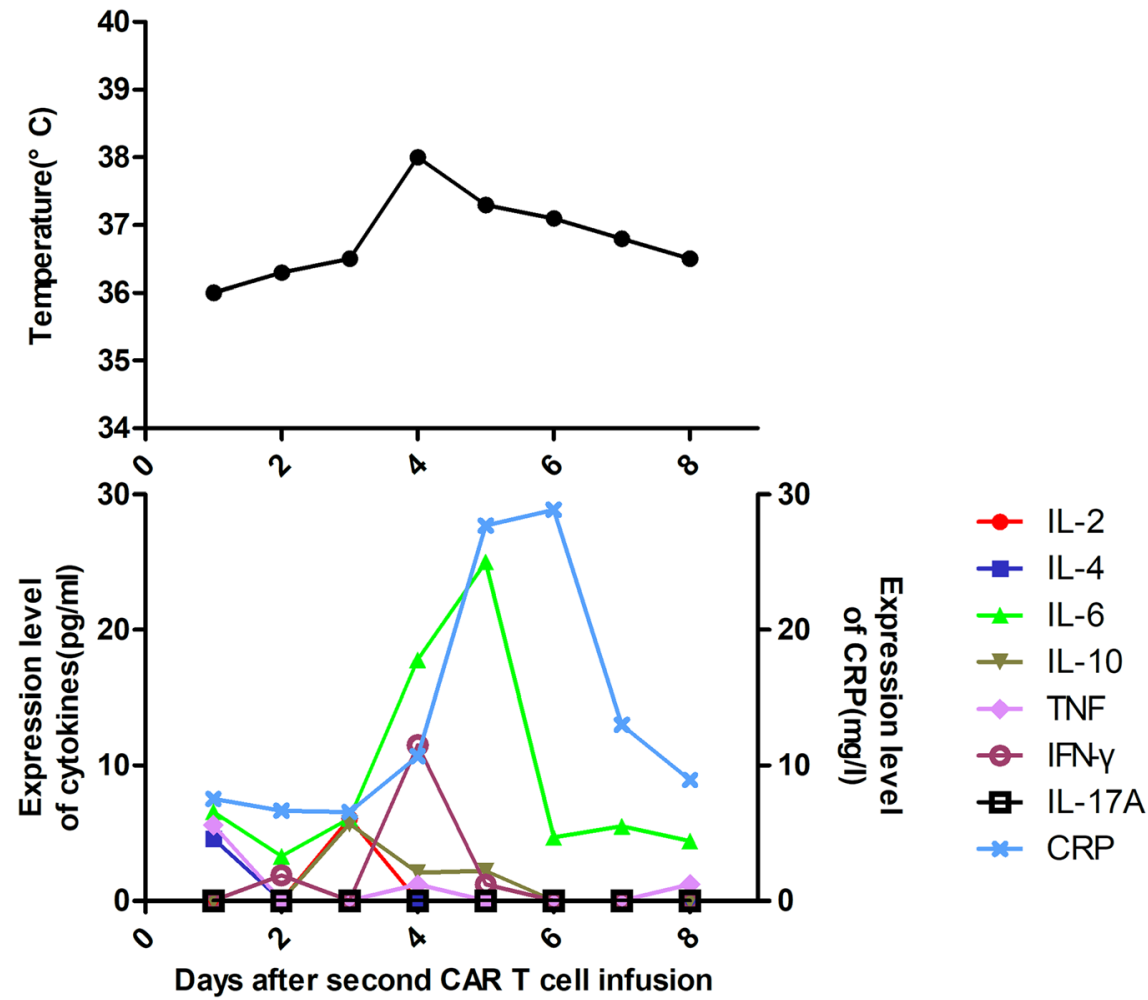

Fig. 1 Temperature, serum levels of cytokines and CRP of the patient after CAR T cells infusions. a After the first CAR T cells infusion. $\mathbf{b}$ After the second CAR T cells infusion 
a

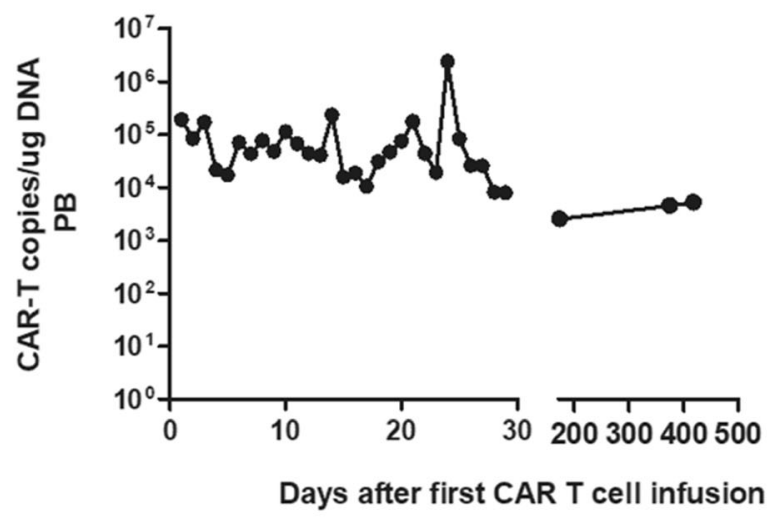

b

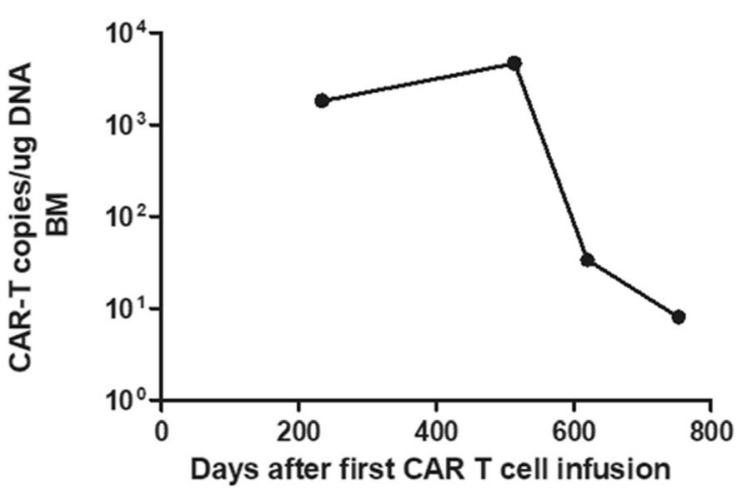

C

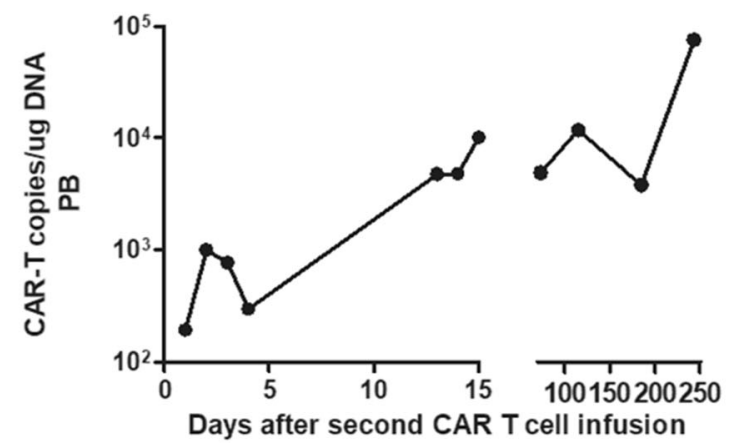

Fig. 2 Copies of CD19-specific CAR-T cells in the peripheral blood/bone marrow of the patient after CAR-T cells infusions. Abbreviation: PB, peripheral blood; BM, bone marrow. a. CD19-CAR T cell copies from peripheral blood mononuclear cells after the first infusion. b. CD19-CAR-T cell copies from bone marrow mononuclear cells after the first infusion. c.CD19-CAR-T cell copies from peripheral blood mononuclear cells after the second infusion

still attained morphological remission and was negative for MRD detected by flow cytometry analysis $(0.0 \times$ $10^{-4}$ ), with complete donor chimerism. Unfortunately, the patient did not undergo a bone marrow examination again during follow-up. Five months later, morphological relapse occurred with $38 \%$ blast cells. A positive MRD (27.8\%) and SET-NUP214 transcript (231.15\%) were detected, accompanied by an extremely low number of DNA copies of anti-CD19 CAR-T cells (8.05 copies per microgram) in the bone marrow specimen (Fig. 2b) and $68.7 \%$ donor cell chimerism. The patient was again treated with idarubicin, vincristine, and dexamethasone as a reinduction chemotherapy. However, flow cytometry analysis revealed $45.5 \%$ of blast cells with a phenotype of B-ALL (with expression of CD7, CD34, HLA-DR, CD19, CD33, CD38, cCD79a, CD71, and CD22) after chemotherapy, and the bone marrow short tandem repeat dropped to $55.5 \%$. The patient received a second infusion of donor-derived humanized CD19-directed CAR-T cells, which has the same costimulatory domain and coactivated domain as mouse-derived CAR-19, except that in the scFv domain of CAR, the humanized CAR-19 recognition site is HD37.The total dosage of humanized CD19-directed CAR-T cells was $1 \times 10^{7} / \mathrm{kg}$ (transduction efficiency $42 \%$ ). The same lympho-depletion regimen of FC (fludarabine /cyclophosphamide) conducted before CAR-T cell infusion was administered. The patient developed a temporary low fever on the first day after infusion, for which nonsteroidal anti-inflammatory drugs were effective. Cytokine and CRP levels increased slightly, indicating grade 1 CRS (Fig. 1b). The level and duration of fever and the increase in cytokine and CRP levels were lower than the previously observed degree after the first CAR-T cell infusion. Moreover, new-onset acute GvHD did not occur after the second CAR-T cell infusion. CR, 99\% donor chimerism, and significantly increased expansion and persistence of CD19-directed CAR-T cells (Fig. 2c) were achieved after infusion. The patient was still alive at the time of the 8 month follow-up evaluation. 


\section{Discussion}

MPAL is considered a high-risk leukemia with a dismal prognosis, and patients should be considered candidates for post-remission consolidation with HSCT, in either CR1 or CR2, particularly patients who are consecutively positive for MRD [9-12]. Patients with MPAL who are undergoing HSCT might achieve similar results compared with those with AML or ALL; however, a population of patients experience relapse after HSCT. PostHSCT relapse is generally associated with a grave prognosis and is the primary cause of death for many of these patients. Promising clinical outcomes have been reported in refractory or relapsed B-cell malignancies, including patients with ALL and diffuse large B-cell lymphoma treated with CD19-targeted CAR-T cells [13-17]. Compared with autologous CAR-T cell therapy, allogeneic CAR-T cells have more advantages. They are free of contamination from inadvertently transduced leukemic blasts, have a lower incidence of $\mathrm{T}$ cell dysfunction, are easier to harvest, and are more tolerated of ex vivo manipulation. Moreover, allogeneic CAR-T cells can be prepared in advance and kept in reserve for when they are needed, reducing the waiting time for relapsed patients. Donor-derived CD19-targeted CAR-T cell therapy might be a promising therapeutic option for relapsed or refractory MPAL after HSCT, as more than two-thirds of patients with MPAL express B-lineage antigens on blasts. However, durable remission after CD19targeted immunotherapy remains a major challenge. A large proportion of CR patients relapse within 1 year $[18,19]$. Several assumptions have been made regarding the causes of recurrence, including depletion of CAR-T cells, abnormal function of CAR-T cells induced by the microenvironment or other factors, and a loss or diminished expression of targeting antigens on the cell surface [20-25].

In our case, a patient with a consecutively positive MRD pre-HSCT quickly relapsed within 6 months after HSCT. He then successfully achieved CMR after CD19targeted CAR-T cell therapy derived from the same donor. Unfortunately, he relapsed again when extremely low numbers of DNA copies of CD19-modified CAR-T cells were detected in his bone marrow 2 years after the first infusion, and the blasts remained CD19-positive. Obviously, although CAR-T cells persisted for a relatively long time in this case, CAR-T cell depletion eventually occurred. However, the question of how to extend CAR-T cell persistence in vivo has still not been answered satisfactorily, and there is no optimal treatment guideline for recurrence after CD19-modified CAR-T cell therapy. Recent studies have confirmed that the host's immune response can recognize epitopes of murine $\mathrm{ScFv}$ domains of the previous CAR structure, resulting in invalidation of subsequent infusions [26]. Therefore, we chose to treat donor-derived humanized CD19 CAR-T cells and induced a second CMR without severe CRS and acute GvHD. Additionally, the patient maintained continuous CMR, and the persistence of CD19-directed CAR-T cells was detected during the 8 month follow-up period. To address the possible causes of recurrence after CAR-T cell treatment and to improve long-term outcome, a cohort of studies and clinical trials have been conducted. These involved dual-signaling CAR-T cell therapies and preventive infusion of a second CAR-T cell product targeting a different antigen when a significant and sustained decline of copies of previous CAR-T cells was detected before the possibility of relapse [27-29].

\section{Conclusion}

Our case demonstrates the efficacy and safety of humanized donor-derived CD19-modified CAR-T cells infusion for treating recurrent MPAL, which was previously exposed to murine-derived CD19-CAR-T cells. Further verification is needed to evaluate this strategy.

\section{Abbreviations \\ MPAL: mixed phenotype acute leukemia; ALL: acute lymphoblastic leukemia; AML: acute myelocytic leukemia; HSCT: hematopoietic stem cell transplantation; CAR-T: chimeric antigen receptor T; sCFvs: single-chain variable fragment; CR: complete remission; CMR: complete molecular remission; MRD: minimal residual disease; CRP: C-reactive protein; CRS: cytokine release syndrome; GvHD: graft-versus-host disease}

\section{Acknowledgements}

The authors thank to all members of the study team, the patient and his family, and Shanghai Uni-CAR Technology Co., Ltd.

\section{Authors' contributions}

$M L, Z L, M H$ and $L K$ were responsible of the clinical management of the patient presented and acquisition of data, and helped to draft the manuscript. HQ, JZ, DW were responsible of the clinical management, interpretation of the data, and drafting the manuscript. XW, QC and XK collected and analyzed data. The author(s) read and approved the final manuscript.

\section{Funding}

Funded by the Jiangsu Provincial Key Medical Center (YXZXA2016002), the Priority Academic Program Development of Jiangsu Higher Education Institutions (PAPD), and National Natural Science Foundation of Jiangsu Province (SBE2018740700)

\section{Availability of data and materials}

Data sharing is not applicable to this article as no datasets were generated or analysed during the current study.

\section{Ethics approval and consent to participate}

The study was approved by the Institutional the First Affiliated Hospital of Soochow University. The patients consented to participate.

\section{Consent for publication}

Written informed consent for research and publication from the patients was obtained.

Competing interests

The authors declare that they have no competing interests. 


\section{Author details}

'Jiangsu Institute of Hematology, National Clinical Research Center for Hematologic Diseases, Collaborative Innovation Center of Hematology, The First Affiliated Hospital of Soochow University, Institute of Blood and Marrow Transplantation, Soochow University, 188 Shizi Street, Suzhou 215006, P.R. China. ${ }^{2}$ Suzhou Yongding Hospital, Suzhou, People's Republic of China. ${ }^{3}$ Institute of Biomedical Engineering and Technology, Shanghai Engineering Research Center of Molecular Therapeutics and New Drug Development, School of Chemistry and Molecular Engineering, East China Normal University, NO, 3663 North Zhongshan Road, Shanghai 200065, China. ${ }^{4}$ Shanghai Unicar-Therapy Bio-medicine Technology Co., Ltd, No 1525 Minqiang Road, Shanghai 201612, China.

Received: 22 April 2020 Accepted: 13 August 2020

Published online: 31 August 2020

\section{References}

1. Weinberg OK, Arber DA. Mixed-phenotype acute leukemia: historical overview and a new definition. Leukemia. 2010;24(11):1844-51.

2. van den Ancker $\mathbf{W}$, Terwijn $M$, et al. Acute leukemias of ambiguous lineage: diagnostic consequences of the WHO2008 classification. Leukemia. 2010; 24(7):1392-6.

3. Yan L, Ping N, et al. Clinical, immunophenotypic, cytogenetic, and molecular genetic features in 117 adult patients with mixed-phenotype acute leukemia defined by WHO-2008 classification. Haematologica. 2012;97(11): 1708-12.

4. Maruffi M, Sposto R, et al. Therapy for children and adults with mixed phenotype acute leukemia: a systematic review and meta-analysis. Leukemia. 2018;32(7):1515-28.

5. Matutes E, Pickl WF, et al. Mixed-phenotype acute leukemia: clinical and laboratory features and outcome in 100 patients defined according to the WHO 2008 classification. Blood. 2011;117(11):3163-71.

6. Rubnitz JE, Onciu M, et al. Acute mixed lineage leukemia in children: the experience of St Jude Children's research hospital. Blood. 2009;113(21): 5083-9.

7. Aribi A, Bueso-Ramos C, et al. Biphenotypic acute leukaemia: a case series. Br J Haematol. 2007;138(2):213-6.

8. Zheng $\mathrm{C}, \mathrm{Wu} \mathrm{J}$, et al. What is the optimal treatment for biphenotypic acute leukemia. Haematologica. 2009;94(12):1778-80 author reply 1780.

9. Tian $\mathrm{H}, \mathrm{Xu}$ Y, et al. Comparison of outcomes in mixed phenotype acute leukemia patients treated with chemotherapy and stem cell transplantation versus chemotherapy alone. Leuk Res. 2016;45:40-6.

10. Shimizu $H$, Saitoh $T$, et al. Allogeneic hematopoietic stem cell transplantation for adult patients with mixed phenotype acute leukemia: results of a matched-pair analysis. Eur J Haematol. 2015;95(5):455-60

11. Munker R, Labopin M, et al. Mixed phenotype acute leukemia: outcomes with allogeneic stem cell transplantation. A retrospective study from the acute leukemia working party of the EBMT. Haematologica. 2017;102(12): 2134-40.

12. Munker R, Brazauskas $R$, et al. Allogeneic hematopoietic cell transplantation for patients with mixed phenotype acute leukemia. Biol Blood Marrow Transplant. 2016;22(6):1024-9.

13. Sadelain $M$, Brentjens $R$, et al. The basic principles of chimeric antigen receptor design. Cancer Discov. 2013;3(4):388-98.

14. Gauthier J, Yakoub-Agha I. Chimeric antigen-receptor T-cell therapy for hematological malignancies and solid tumors: clinical data to date, current limitations and perspectives. Curr Res Transl Med. 2017;65(3):93-102.

15. Ghobadi A. Chimeric antigen receptor T cell therapy for non-Hodgkin lymphoma. Curr Res Transl Med. 2018;66(2):43-9.

16. Grupp S. Beginning the CAR T cell therapy revolution in the US and EU. Curr Res Transl Med. 2018;66(2):62-4.

17. Quesnel B. CAR T-cells: a John von Neumann legacy. Curr Res Transl Med. 2018;66(2):35-6.

18. Kenderian SS, Porter DL, et al. Chimeric antigen receptor $\mathrm{T}$ cells and hematopoietic cell transplantation: how not to put the CART before the horse. Biol Blood Marrow Transplant. 2017;23(2):235-46.

19. Lee DW, Kochenderfer JN, et al. T cells expressing CD19 chimeric antigen receptors for acute lymphoblastic leukaemia in children and young adults: a phase 1 dose-escalation trial. Lancet. 2015;385(9967):517-28.

20. Grupp SA, Kalos M, et al. Chimeric antigen receptor-modified T cells for acute lymphoid leukemia. N Engl J Med. 2013;368(16):1509-18.
21. Maude SL, Teachey DT, et al. CD19-targeted chimeric antigen receptor T-cell therapy for acute lymphoblastic leukemia. Blood. 2015;125(26):4017-23.

22. Sotillo E, Barrett DM, et al. Convergence of acquired mutations and alternative splicing of CD19 enables resistance to CART-19 immunotherapy. Cancer Discov. 2015;5(12):1282-95.

23. Ruella M, Barrett DM, et al. Dual CD19 and CD123 targeting prevents antigen-loss relapses after CD19-directed immunotherapies. J Clin Invest. 2016;126(10):3814-26.

24. Ruella M, Maus MV. Catch me if you can: leukemia escape after CD19directed T cell immunotherapies. Comput Struct Biotechnol J. 2016;14:35762.

25. Maude SL, Frey N, et al. Chimeric antigen receptor T cells for sustained remissions in leukemia. N Engl J Med. 2014;371(16):1507-17.

26. Turtle CJ, Hanafi LA, et al. CD19 CAR-T cells of defined CD4+:CD8+ composition in adult B cell ALL patients. J Clin Invest. 2016;126(6):2123-38.

27. Zhao J, Song Y, et al. Clinical trials of dual-target CAR T cells, donor-derived CAR T cells, and universal CAR T cells for acute lymphoid leukemia. J Hematol Oncol. 2019;12(1):17.

28. Wang N, Hu X, et al. Efficacy and safety of CAR19/22 T-cell cocktail therapy in patients with refractory/relapsed B-cell malignancies. Blood. 2020;135(1): $17-27$.

29. Pan J, Zuo S, et al. Sequential CD19-22 CAR T therapy induces sustained remission in children with r/r B-ALL. Blood. 2020;135(5):387-91.

\section{Publisher's Note}

Springer Nature remains neutral with regard to jurisdictional claims in published maps and institutional affiliations.
Ready to submit your research? Choose BMC and benefit from:

- fast, convenient online submission

- thorough peer review by experienced researchers in your field

- rapid publication on acceptance

- support for research data, including large and complex data types

- gold Open Access which fosters wider collaboration and increased citations

- maximum visibility for your research: over $100 \mathrm{M}$ website views per year

At $\mathrm{BMC}$, research is always in progress.

Learn more biomedcentral.com/submissions 